\title{
Combining Power Control and Blind Equalization in Wireless Networks
}

\author{
Kirankumar Vissa, K. J. Ray Liu \\ Inst. for Sys. Research and Elec. Engg. Department \\ University of Maryland \\ College Park, MD 20742 \\ (vissa, kjrliu@isr.umd.edu)
}

\author{
Farrokh Rashid-Farrokhi \\ Wireless Communications Research Dept. \\ Bell Labs, Lucent Technologies \\ 791 Holmdel-Keyport Rd, Rm. R-111 \\ Holmdel, NJ 07733
}

\begin{abstract}
Decentralized power control techniques achieve a target SINR (Signal to Interference-Noise Ratio) in wireless networks assuming knowledge of link gains and measurement of interference powers. In this paper, we propose a joint power control-blind equalization algorithm that redefines the power control problem and, importantly, does not need measurement of link gains and interference powers at the base station or sending training sequence. We consider uplink transmission with multiple reception at each station.

Convergence of the algorithm for low delay spread channels is shown theoretically and verified by simulation. The proposed scheme also enables users to achieve a desired bit error rate based directly on a selectable threshold. In the traditional scenario, an error in the assumed channel and array response causes a steep degradation in performance while our algorithm does not degrade though there is error in the estimated response from blind equalization.
\end{abstract}

\section{Introduction}

One of the main factors that degrades performance in wireless communications is the co-channel interference. Power Control is one direct approach towards minimizing co-channel interference. In power control $[1,3,7]$, the transmitter powers are continually adjusted depending on the Signal to Interference-Noise Ratio (SINR), so that the quality of the weak links is improved and a minimum level performance is met by all the links.

Blind equalization is used to equalize the distortion produced by the communication channel and thereby estimate the transmitted symbols, without the use of training symbols. It is especially useful in wireless communication with rapidly changing environment $[6,5]$. Since blind equalization algorithms inherently try to maximize the carrier-tointerference ratio at the output of the equalizer, it is worth considering a method of combining both power control and blind equalization so that they work in tandem.

Current methods for power control implement a distributed algorithm [7], [3], [1] which has the disadvantage that it assumes the knowledge of link gains and interference power measurement at the base station that is not easily available in practice. Recently, algorithms combining power control with beamforming and base station assignment have been proposed based on the same assumptions [4]. In this paper, we present an approach that combines blind equalization and power control. We redefine the power control problem and base our power updates solely on the information directly available from the blind equalization algorithm. The advantage is that we do not need the knowledge of link gains and additional measurement of interference powers.

Convergence properties for a low delay-spread channel are discussed. Simulation results illustrating the performance and comparision with the joint power controlbeamforming algorithm are also presented.

\section{Problem Formulation}

\subsection{Decentralized Power Control}

A set of $M$ transmitter-reciever pairs which use the same frequency channel is considered. With link gains $\left\{G_{i j}\right\}$, transmitter powers and noise powers $\left\{P_{i}\right\}$ and $\left\{N_{i}\right\}$, the Signal to Interference-Noise Ratio (SINR) at receiver $i$ is given by

$$
\Gamma_{i}=\frac{G_{i i} P i}{\sum_{j \neq i} G_{j i} P j+N_{i}}
$$

The power control problem to achieve a desired threshold $\gamma_{0}$ is defined as

$$
\begin{array}{ll}
\operatorname{minimize} & \sum_{i} P_{i} \\
\text { subject to } & \Gamma_{i} \geq \gamma_{0}, \quad 1 \leq i \leq M
\end{array}
$$


The constraint can be expressed in matrix form as [I $\left.\gamma_{0} \mathbf{F}\right] \mathbf{P} \geq \mathbf{u}$ where

$$
\begin{aligned}
& u_{i}=\frac{\gamma_{0} N_{i}}{G_{i i}}, \quad 1 \leq i \leq M \quad \text { and } \\
& F_{i j}=\left\{\begin{array}{ccccc}
0 & & \text { if } & j=i \\
\frac{G_{j i}}{G_{i i}}> & 0 & \text { if } & j \neq i
\end{array}\right.
\end{aligned}
$$

It can be shown $[3,7,2]$ that if the spectral radius of $\mathrm{F}$ is less than $\frac{1}{\gamma_{0}}$, the optimal power vector exists and the following decentralized iteration converges to the optimal power vector $\hat{\mathbf{P}}=\left[I-\gamma_{0} F\right]^{-1} \mathbf{u}$.

$$
\mathbf{P}^{n+1}=\gamma_{0} F \mathbf{P}^{n}+\mathbf{u}
$$

At each iteration, transmitters update their powers based on the measured interference power measured at the receivers and the link gain information transmitted on the control channel.

\subsection{Blind Equalization - ILSP Algorithm}

We use the Iterative Least Squares Projection (ILSP) algorithm proposed in [6]. This algorithm gives us a good formulation to incorporate power control especially because a direct analytic relationship exists for the symbol detection error probability. The system consists of $M$ base stations, each with $d$ co-channel mobile transmitters. Each source generates binary data $s_{k}(n)$ with amplitude $p_{k}$ transmitted over a multi-path channel $\mathbf{a}_{k}$, which is composed of the attenuation, phase-lag and the array response at the receiver antenna for each path. The antenna output at receiver with noise $\mathbf{v}(n)$ is given by

$$
\mathbf{x}(n)=\sum_{k=1}^{d} \mathbf{a}_{k} p_{k} s_{k}(n)+\mathbf{v}(n)
$$

Here we adopt a low delay-spread multi-path channel. ILSP works on blocks of data of size $\mathrm{N}$ with a shifting window. We define $\mathbf{X}^{\mathbf{i}}=[\mathbf{x}(i N+1) \mathbf{x}(i N+2) \cdots \mathbf{x}((i+1) N)]$, $\mathbf{S}^{\mathbf{i}}=[\mathbf{s}(i N+1) \mathbf{s}(i N+2) \cdots \mathbf{s}((i+1) N)]$ and $\mathbf{V}^{\mathbf{i}}=$ $[\mathbf{v}(i N+1) \cdots \mathbf{v}((i+1) N)]$. To simplify notation, we use $\mathbf{X} \equiv \mathbf{X}^{\mathbf{i}}$ etc., and get the relation

$$
\mathbf{X}=\mathbf{A S}+\mathbf{V}
$$

where $\mathbf{A}=\left[\begin{array}{llll}p_{1} \mathbf{a}_{1} & p_{2} \mathbf{a}_{2} & \cdots & p_{d} \mathbf{a}_{d}\end{array}\right]$. The ILSP algorithm makes use of the finite alphabet property of the input to implement a least squares algorithm that has good convergence properties for channels with low delay spread. Given $\mathbf{A}_{0}$, the following iteration is performed till convergence:
$\overline{\mathbf{S}}_{m}=\mathbf{A}_{m-1}^{\dagger} \mathbf{X}$ where $\mathbf{A}_{m-1}^{\dagger}=\left(\mathbf{A}_{m-1}^{*} \mathbf{A}_{m-1}\right)^{-1} \mathbf{A}_{m-1}^{*}$

$\mathbf{S}_{m}=\operatorname{proj}\left[\overline{\mathbf{S}}_{m}\right]$ (projection onto finite alphabet)

$\mathbf{A}_{m}=\mathbf{X} \mathbf{S}_{m}^{\dagger}$ where $\mathbf{S}_{m}^{\dagger}=\mathbf{S}_{m}^{*}\left(\mathbf{S}_{m} \mathbf{S}_{m}^{*}\right)^{-1}$

It is shown in $[6,5]$ that each element $\bar{s}_{k}(n)$ is normally distributed with

$$
E\left[\bar{s}_{k}(n)\right]=s_{k}(n) \text { and } \operatorname{Var}\left[\bar{s}_{k}(n)\right]=2 \sigma^{2}\left(\mathbf{A}^{H} \mathbf{A}\right)_{k k}^{-1}
$$

The probability of wrong symbol detection is given by

$$
\operatorname{Pr}_{k}\left(s^{(i)}, s^{(j)}\right)=Q\left(\frac{d_{i, j}}{2 \sqrt{\operatorname{Var}\left[\bar{s}_{k}(n)\right]}}\right)
$$

\section{Combining Blind Equalization with Power Control}

\subsection{Basic Intuition and Redifining the Power con- trol Problem}

To simply understand how to combine the ILSP algorithm with power control, let us reduce the model to the scenario for power control in Section 2.1. This corresponds to the case $d=1$ where there is only one co-channel source for every base station. For example, at the $i$ th base station, $\mathbf{x}_{\mathbf{i}}(n)=p \mathbf{a}_{\mathbf{i}} s_{i}(n)+\mathbf{v}_{\mathbf{i}}(n)$. Here, $\mathbf{v}_{\mathbf{i}}(n)$ is the sum of interference signals from other mobiles, and with a large number of interferers, can be considered Gaussian by the Central Limit Theorem. The power in $\mathbf{v}_{\mathbf{i}}(n)$ is given by $\sigma_{i}^{2}=\sum_{j \neq i} G_{j i} P_{j}+N_{i}$.

From the preceding analysis of (5), we have in this case,

$$
\operatorname{Var}\left[\bar{s}_{i}(n)\right]=2 \sigma_{i}^{2}\left(p_{i}^{2} \mathbf{a}_{i}^{H} \mathbf{a}_{i}\right)^{-1}=2 \frac{\sum_{j \neq i} G_{j i} P_{j}+N_{i}}{G_{i i} P_{i}}
$$

We can see that the expression in (7) is the reciprocal of $\Gamma_{i}$ in Equation (1). Furthermore, $\operatorname{Var}\left[\bar{s}_{i}(n)\right]$ is directly related to the error performance and hence is the appropriate quantity to optimize. The power control problem can now be redefined as follows,

$$
\begin{array}{ll}
\operatorname{minimize} & \sum P_{i}, \\
\text { subject to } & \operatorname{Var}\left(\bar{s}_{i}\right)<\operatorname{var}_{0} \text { for all } i
\end{array}
$$

This definition of the problem holds for the case of multiple co-channel mobiles as well. The joint algorithm listed in Table 1 solves the above power control problem with blind equalization.

\subsection{Analysis and Convergence of the Algorithm}

We present an outline of the proof of convergence considering the general case of multiple co-channel mobiles for 
1. Given $\mathbf{P}(0), n=0, \mathbf{A}_{0}$ and $v a r_{0}$

2. $m=0$. Received data block $\mathbf{X}^{\mathbf{n}}$. Denote $\mathbf{X} \equiv \mathbf{X}^{\mathbf{n}}$.

At each base station take $\mathbf{A}_{0}$ and do:

$$
\begin{aligned}
& \text { i. } m=m+1 \\
& \overline{\mathbf{S}}_{m}=\mathbf{A}_{m-1}^{\dagger} \mathbf{X} \text { where } \mathbf{A}_{m-1}^{\dagger}=\left(\mathbf{A}_{m-1}^{*} \mathbf{A}_{m-1}\right)^{-1} \mathbf{A}_{m-1}^{*} \\
& \mathbf{S}_{m}=\text { proj }\left[\overline{\mathbf{S}}_{m}\right] \\
& \quad \mathbf{A}_{m}=\mathbf{X} \mathbf{S}_{m}^{\dagger} \text { where } \mathbf{S}_{m}^{\dagger}=\mathbf{S}_{m}^{*}\left(\mathbf{S}_{m} \mathbf{S}_{m}^{*}\right)^{-1} \\
& \text { ii. Repeat i until }\left(\mathbf{A}_{m}, \mathbf{S}_{m}\right)=\left(\mathbf{A}_{m-1}, \mathbf{S}_{m-1}\right) \\
& \text { iii. For each mobile } k, \operatorname{var}_{k}=\frac{\left|\mathbf{s}_{\mathbf{m}, \mathbf{k}}-\overline{\mathbf{s}}_{m, k}\right|^{2}}{N} \\
& \text { iv. At each mobile } k, P_{k}(n+1)=\frac{v a r_{k}}{\operatorname{var}_{0}} P_{k}(n) \\
& \text { v. } \mathbf{A}_{0}=\mathbf{A}_{m} \\
& \text { 3. } n=n+1 \text {. Go to Step 2; repeat until } \mathbf{P}(n)=\mathbf{P}(n-1)
\end{aligned}
$$

\section{Table 1. Joint Power Control and Blind Equal- ization}

every base station. Firstly, the expression in (5) for $\operatorname{Var}\left(\bar{s}_{i}\right)$ is simplified as:

$$
\begin{aligned}
\operatorname{Var}\left[\bar{s}_{i}(n)\right] & =2 \sigma_{i}^{2}\left(\mathbf{A}^{H} \mathbf{A}\right)_{i i}^{-1} \\
& =2 \frac{\sum_{j \notin \zeta(i)} G_{j i} P_{j}(n)+N_{i}}{G_{i i} P_{i}(n)} f_{i}\left(\left\{\mathbf{a}_{j}\right\}_{j \in \zeta(i)}\right)
\end{aligned}
$$

where $\zeta(i)$ is the set of all mobiles withinthe same cell as mobile $i$.

The key result is that $f_{i}($.$) is independent of \left\{p_{i}\right\}$ and hence the transmit powers of mobiles in the same cell. Now, define a matrix $\mathbf{F}$ where

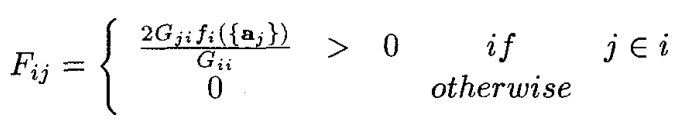

Following (9), the power update step $\mathbf{3}$ in our algorithm of Table 1 can be written as

$$
\begin{aligned}
P_{i}(n+1) & =\frac{2}{v a r_{0}} \frac{\sum_{j \in \zeta(i)} G_{j i} P_{j}(n)+N_{i}}{G_{i i} P_{i}(n)} f_{i}\left(\left\{\mathbf{a}_{j}\right\}\right) P_{i}(n) \\
& =\frac{2 f_{i}\left(\left\{\mathbf{a}_{j}\right\}\right)}{\operatorname{var}_{0}} \frac{\sum_{j \in \zeta(i)} G_{j i} P_{j}(n)+N_{i}}{G_{i i}}
\end{aligned}
$$

Considering the whole network, Equation (11) can be written in matrix form as

$$
\mathbf{P}(n+1)=\frac{1}{v a r_{0}} \mathbf{F P}(n)+\mathbf{u}
$$

where $u_{i}=\frac{1}{v a r_{0}} \frac{N_{i}}{G_{i i}}$. This formulation is similar to (3) and convergence is established following the proof in [1],[2]. An optimum power vector exists and the power update converges if $\rho(\mathbf{F})<v a r_{0}$. The role of the blind equalization is to provide, for every iteration of power update, the appropriate value of $\operatorname{var}(n)$

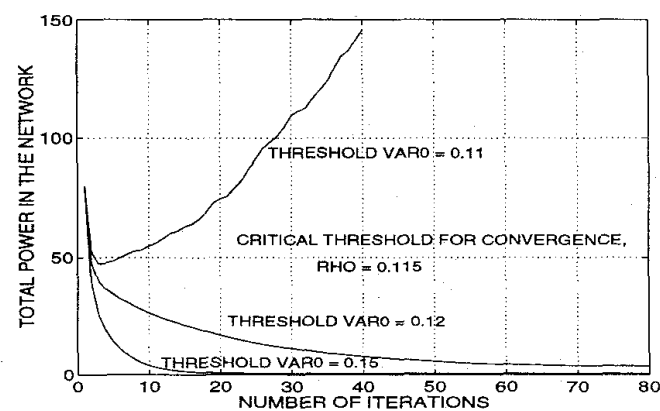

Figure 1. Convergence of our Joint Algorithm for different thresholds

\section{Simulations and Performance}

Performance study is done by simulation of a wireless TDMA network having 20 base stations and 80 mobiles. Each mobile transmits PAM data over multi-path channels with upto 4 paths, and each base station employs an antenna array with 6 elements. The equalization on blocks of 1000 data symbols, and distributed power updates are jointly performed using the proposed algorithm.

Figure 1 shows the convergence of the total power in the network for different thresholds. The critical threshold is $\rho(F)=0.115$. The algorithm converges when threshold $v a r_{0}<\rho(F)$ which confirms the theoretical result in Section 3.2. The convergence is faster as $v a r_{0}$ increases. Figure 2 shows, for a typical threshold $\operatorname{var} 0=0.12$, how the bit error rate decreases by nearly 40 times while the total power also converges to a low value. Then, we keep the total power constant and check the error rate if power control were absent; we find that power control has improved the $\mathrm{BER}$ of the equalization from $1.2 \mathrm{e}-03$ to $2.5 \mathrm{e}-05$.

Figure 3 shows the comparision with the results of the joint power control and beamforming (PC-MVDR) algorithm proposed in [4], which is run in the same network. Beamforming assumes knowledge of the channel responses and hence is an idealized scenario whereas our joint algorithm (PC-BE) is blind to the channel response. To make a fair comparision, we find the magnitude of error in the channel estimated through equalization after convergence and introduce an error of same magnitude into the channel response assumed for MVDR. Figure 3 shows that the beamforming algorithm has a steep performance decline with a channel mismatch whereas our algorithm, under the same conditions performs an order of magnitude better and closely approaches the ideal scenario of PC-MVDR with perfect channel match. 


\section{Conclusions}

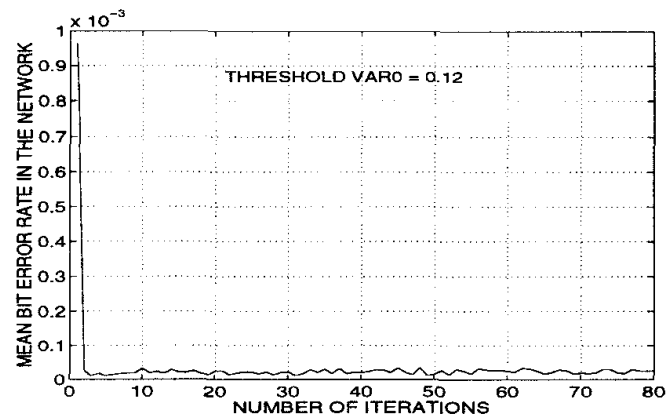

Figure 2. Decline in Bit Error Rate for our Joint Algorithm

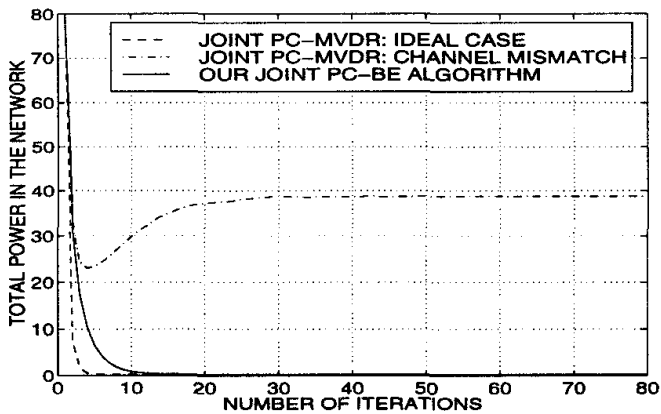

Figure 3. Comparision with joint PC-MVDR algorithm
We have proposed a combined power control and blind equalization algorithm which redefines the power control problem in terms of a quantity directly related to the error performance of the equalization. Furthermore, the algorithm does not require additional link gain information or measurement of interference power, but rather uses information directly available from the blind equalization. Theoretical result for the convergence of the algorithm is obtained and is supported by simulation results. Performance compares favorably with the joint PC-MVDR algorithm.

Future research could extend this approach to channels with high delay spread. Further, the power control problem can be combined with other blind equalization algorithms as well by redifining the power control problem, provided we find suitable parameters from equalization.

\section{References}

[1] G. Foschini. A simple distributed autonomous power control algorithm and its convergence. IEEE Trans. Vehicular Technol., November, 1993.

[2] F. R. Gantmacher. The Theory of Matrices. Chelsea, New York, third edition, 1990.

[3] S. A. Grandhi, R. Vijayan, and D. J. Goodman. Distributed power control in cellular radio systems. IEEE Trans. Communications, pages 226-8, February-April, 1994.

[4] F. Rashid-Farrokhi, L. Tassiulas, and K. J. R. Liu. Joint optimal power control and beamforming in wireless networks using antenna arrays. IEEE Trans. Communications, pages 1313-24, October, 1998.

[5] S. Talwar. Blind separation of co-channel digital signals using antenna arrays. $\mathrm{PhD}$ thesis, $\mathrm{Ph} . \mathrm{D}$. Dissertation, Dept. of Scientific Computing and Computational Mathematics, Stanford University, 1996.

[6] S. Talwar and A. Paulraj. Blind separation of synchronous co-channel digital signals using an antenna array. ii. performance analysis. IEEE Trans. Signal Processing, pages 70618, March, 1997.

[7] J. Zander. Distributed co-channel interference control in cellular radio systems. IEEE Trans. Vehicular Technol, pages 305-11, August, 1992. 